\title{
Chetomin induces degradation of XIAP and enhances TRAIL sensitivity in urogenital cancer cells
}

\author{
KIMIHIRO YANO ${ }^{1,2^{*}}$, MANO HORINAKA ${ }^{1 *}$, TATSUSHI YOSHIDA ${ }^{1}$, TAKASHI YASUDA ${ }^{1,2}$, \\ HIROYA TANIGUCHI $^{1,3}$, AHMED E. GODA ${ }^{1,4}$, MIKI WAKADA ${ }^{1}$, SAE YOSHIKAWA $^{1}$, \\ TERUKAZU NAKAMURA ${ }^{2}$, AKIHIRO KAWAUCHI ${ }^{2}$, TSUNEHARU MIKI $^{2}$ and TOSHIYUKI SAKAI ${ }^{1}$
}

\begin{abstract}
Departments of ${ }^{1}$ Molecular-Targeting Cancer Prevention, ${ }^{2}$ Urology, and ${ }^{3}$ Gastroenterology, Graduate School of Medical Science, Kyoto Prefectural University of Medicine, Kawaramachi-Hirokoji, Kamigyo-ku, Kyoto 602-8566, Japan;

${ }^{4}$ Department of Pharmacology and Toxicology, Faculty of Pharmacy, Tanta University, Tanta, Egypt
\end{abstract}

Received September 15, 2010; Accepted November 2, 2010

DOI: $10.3892 /$ ijo.2010.874

\begin{abstract}
Tumor necrosis factor-related apoptosis-inducing ligand (TRAIL) is one of the most promising anti-cancer agents, but some tumor types develop resistance to TRAIL. Here, we report that chetomin, an inhibitor of hypoxiainducible factors, is a potent enhancer of TRAIL-induced apoptosis. TRAIL or chetomin alone weakly induced apoptosis, but the combination of chetomin and TRAIL synergistically induced apoptosis in prostate cancer PC-3 cells. The combination of chetomin and TRAIL induces the activation of caspase-3, -8, -9 and -10 . Among the apoptotic factors related to the TRAIL pathway, chetomin markedly decreased the X-linked inhibitor of apoptosis (XIAP) protein levels in a dose-dependent manner, but other IAP family members, TRAIL receptors and Bcl-2 family members were not altered by chetomin. Using XIAP siRNA instead of chetomin, down-regulation of XIAP sensitized PC-3 cells to TRAIL-induced apoptosis. Conversely, transient transfection of XIAP reduced the apoptotic response to combined treatment with chetomin and TRAIL. Treatment with chetomin induced a rapid decrease in XIAP protein levels but had no effect on XIAP mRNA levels. Since chetomin-mediated XIAP
\end{abstract}

Correspondence to: Dr Toshiyuki Sakai, Department of Molecular-Targeting Cancer Prevention, Graduate School of Medical Science, Kyoto Prefectural University of Medicine, Kawaramachi-Hirokoji, Kamigyo-ku, Kyoto 602-8566, Japan

E-mail: tsakai@koto.kpu-m.ac.jp

${ }^{*}$ Contributed equally

Abbreviations: XIAP, X-linked inhibitor of apoptosis; TRAIL, tumor necrosis factor-related apoptosis-inducing ligand; PARP, poly (ADP-ribose) polymerase; DR5, death receptor 5; DcR1, decoy receptor 1; BAX, Bcl2-associated X protein; cIAP1, cellular inhibitor of apoptosis 1; PBMC, peripheral blood mononuclear cells; HIF, hypoxia-inducible factor

Key words: chetomin, XIAP, TRAIL, urogenital cancer down-regulation was completely prevented by proteasome inhibitors, it was suggested that chetomin induces the degradation of the XIAP protein in a proteasome-dependent manner. Additionally, chetomin also sensitized renal cancer Caki-1 cells and bladder cancer UM-UC-3 cells to TRAIL-induced apoptosis via down-regulation of XIAP. Co-treatment of chetomin and TRAIL did not enhance apoptosis in normal peripheral blood mononuclear cells (PBMC). Taken together, these findings suggest that TRAIL and chetomin synergistically induce apoptosis in human urogenital cancer cells through a mechanism that involves XIAP down-regulation by chetomin.

\section{Introduction}

The prognoses for urogenital cancer patients with metastatic lesions are poor, even though new regimens of systemic chemotherapy have been introduced in recent years. For example, the combination of docetaxel and prednisone statistically prolonged overall survival and improved the quality of life of prostate cancer patients (1). Therefore, the FDA approved docetaxel for the treatment of metastatic hormonerefractory prostate cancer in 2004. On the other hand, sunitinib (tyrosine kinase inhibitor) and sorafenib (multikinase inhibitor) were approved for the treatment of metastatic renal cell carcinoma patients $(2,3)$. The outcome of these treatments is still insufficient, and new strategies are required to improve metastatic urogenital cancer therapy.

Tumor necrosis factor-related apoptosis-inducing ligand (TRAIL) belongs to the TNF family, including TNF- $\alpha$ and Fas ligand (4). TRAIL induces apoptosis preferentially in cancer cells in vitro and in vivo, but has little or no toxicity to normal cells (5-9). Therefore, TRAIL is an attractive agent for cancer treatment. Human recombinant TRAIL and agonistic antibodies against TRAIL receptors are under going clinical phase I and II trials (10). However, some tumor types develop resistance to TRAIL (11), and it is necessary to overcome this resistance.

X-linked inhibitor of apoptosis (XIAP) inhibits the activation of both initiator caspase- 9 and effector caspase- 3 and -7 by binding to these caspases with its baculovirus IAP repeat domains (12). XIAP is frequently overexpressed in many human cancers, including urogenital cancers, and is involved 
in a worse prognosis or early recurrence (13-15). Many studies showed that overexpression of XIAP in various cancer cell lines augments resistance to chemotherapeutic agents, including TRAIL (16-18). In contrast, down-regulation of XIAP by siRNA or antisense oligonucleotides sensitizes resistant cancer cells to TRAIL-induced apoptosis $(19,20)$. The above evidence indicates that XIAP is one of the targets to enhance TRAIL sensitivity as well as a direct target for cancer treatment.

Chetomin, a dithiodiketopiperazine metabolite of fungus Chaetomium species, disrupts the structure of the $\mathrm{CH} 1$ domain of p300 transcriptional coactivators and interferes with the interaction of hypoxia-inducible factor (HIF)-1 $\alpha$, thereby inhibiting hypoxia-inducible transcription (21). It has also been shown that chetomin markedly reduced colon and prostate tumor xenograft growth. In the search for new strategies to enhance TRAIL sensitivity, we found that chetomin is a potent enhancer of TRAIL-induced apoptosis via the down-regulation of XIAP in human urogenital cancer cells.

\section{Materials and methods}

Reagents. Chetomin and soluble recombinant human TRAIL/ Apo2L were purchased from Alexis (San Diego, CA) and PeproTech (London, UK), respectively. MG132, proteasome inhibitor I and proteasome inhibitor II were purchased from Calbiochem (La Jolla, CA). Recombinant human DR5 (TRAIL-R2)/Fc chimera, and the caspase inhibitors zVADfmk, zDEVD-fmk, zIETD-fmk, zLEHD-fmk and zAEVDfmk were purchased from R\&D Systems (Minneapolis, MN). A variety of 96 compounds (SCADS inhibitor kit II) were provided by the Screening Committee of Anticancer Drugs supported by Grant-in-Aid for Scientific Research on Priority Area Cancer from the Ministry of Education, Culture, Sports, Science and Technology, Japan.

Cell culture. Human prostate cancer PC-3 cells, human renal cancer Caki-1 cells and human bladder cancer UM-UC-3 cells were maintained in RPMI-1640 medium with $10 \%$ fetal bovine serum, $2 \mathrm{mM}$ glutamine, $100 \mathrm{U} / \mathrm{ml}$ penicillin, and $100 \mu \mathrm{g} / \mathrm{ml}$ streptomycin and incubated at $37^{\circ} \mathrm{C}$ in a humidified atmosphere containing $5 \% \mathrm{CO}_{2}$. Normal human peripheral blood mononuclear cells (PBMC) were isolated as previously described (22).

Detection of apoptosis. DNA fragmentation was quantified by the percentage of hypodiploid DNA (sub-G1). PC-3, Caki-1 and UM-UC-3 cells and PBMC were treated with PBS containing $0.1 \%$ Triton X-100. Cells were then treated with RNase A (Sigma, St. Louis, MO) and the nuclei were stained with propidium iodide (Sigma). The DNA content was measured using a FACSCalibur flow cytometer and Cell Quest software (Becton-Dickinson, Franklin Lakes, NJ). For all assays, 10,000 cells were counted. The data were analyzed using Student's t-test. Differences were considered significantly different from controls at $\mathrm{P}<0.05$.

Combination index. We calculated the combination index for TRAIL and chetomin using the CalcuSyn 2.0 software (Biosoft, Great Shelford, UK).
Western blot analysis. Western blot analysis was performed as previously described (22). Rabbit polyclonal antibodies for DR5 and DR4 (Prosci, Poway, CA), caspase-3, cleaved caspase-3, Bcl-xL, cIAP-2 and BAX (Santa Cruz Biotechnology, Santa Cruz, CA), survivin and cIAP-1 (R\&D Systems), and mouse monoclonal antibodies for PARP and Bcl-2 (Santa Cruz Biotechnology), XIAP (R\&D Systems), caspase-8, -9 and -10 antibodies (MBL, Nagoya, Japan) and B-actin antibody (Sigma) were used as the primary antibodies. The signal was detected with an ECL Western blot analysis system (GE Healthcare, Piscataway, NJ).

Determination of TRAIL receptor expression. PC-3 cells were treated with DMSO or $200 \mathrm{nM}$ chetomin for $24 \mathrm{~h}$, and then harvested by short trypsinization. The cells were washed once with ice-cold PBS containing $1 \%$ bovine serum albumin (BSA), and resuspended in $100 \mu \mathrm{l}$ PBS with $1 \%$ BSA. Then, $5 \mu \mathrm{g}$ of PE-labeled anti-TRAIL receptor antibody (DR4, DR5, DcR1, or DcR2; eBioscience, San Diego, CA) were added. To assess non-specific staining, PE-labeled control IgG isotypes (eBioscience) were applied. After 30-min incubation on ice, cells were washed and analyzed using a FACSCalibur flow cytometer.

siRNAs. Non-coding siRNA (23) and XIAP siRNA (19) were synthesized by Sigma. Non-coding siRNA was used as a control. In brief, 1 day prior to transfection, PC-3 cells were seeded without antibiotics at a density of $30-40 \%$. Non-coding or XIAP siRNA was transfected into cells using a modified Oligofectamine protocol (Invitrogen, Carlsbad, CA), in which the volume of Oligofectamine was reduced to one-third of the recommended volume to limit toxic effects. The final concentrations of siRNAs were $100 \mathrm{nM}$. Mock samples were treated with Oligofectamine alone. Twenty-four hours after transfection, cells were treated with or without $5 \mathrm{ng} / \mathrm{ml}$ TRAIL for $24 \mathrm{~h}$, and then harvested.

In HIF- $1 \alpha$ siRNA experiments, Silencer predesigned siRNA (Ambion, Austin, TX) targeting HIF-1 $\alpha$ (ID no.: S6539) or Silencer Negative Control no. 1 siRNA (siControl; ID no. 4390843) was used in knockdown experiments. PC-3 cells were transfected with siRNAs (40 nM) using siPORT NeoFX (Ambion) according to the manufacturer's recommendations. At $43 \mathrm{~h}$ post-transfection, the cells were incubated with $100 \mu \mathrm{M}$ of $\mathrm{CoCl}_{2}$ in complete medium for $5 \mathrm{~h}$.

Plasmid DNA transfection. The pCMV6-XL5 control and pCMV6-XL5/XIAP plasmid constructs were purchased from OriGene Technologies (Rockville, MD). In brief, 1 day prior to transfection, PC-3 cells were seeded in 6-well plates without antibiotics at a density of $80 \%$. Plasmid DNA $(2 \mu \mathrm{g})$ was transfected into cells using HilyMax transfection reagent (Dojindo Laboratories, Kumamoto, Japan) according to the manufacturer's instructions. Twenty-four hours after transfection, the medium was replaced with fresh medium, and cells were treated with $200 \mathrm{nM}$ chetomin and/or $5 \mathrm{ng} / \mathrm{ml}$ TRAIL for $24 \mathrm{~h}$, and then harvested.

RNA isolation and quantitative real-time RT-PCR. Total RNA from PC-3 cells was isolated using the RNeasy Plus mini kit (Qiagen, Hilden, Germany) according to the manufacturer's 
A

B

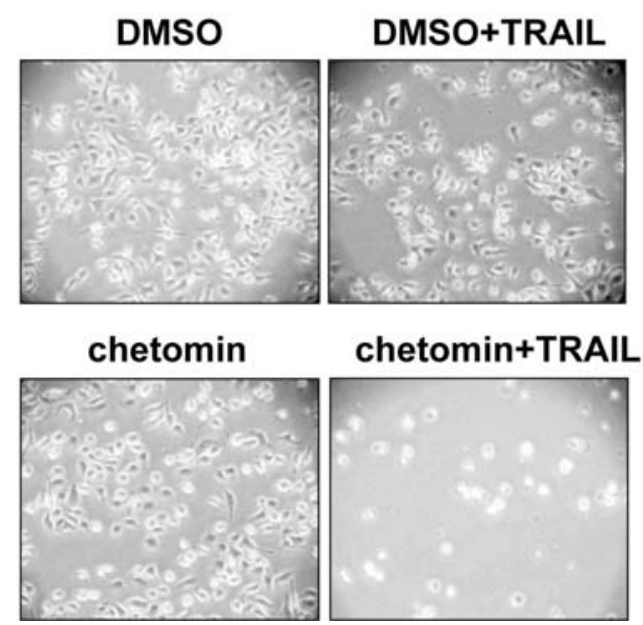

C
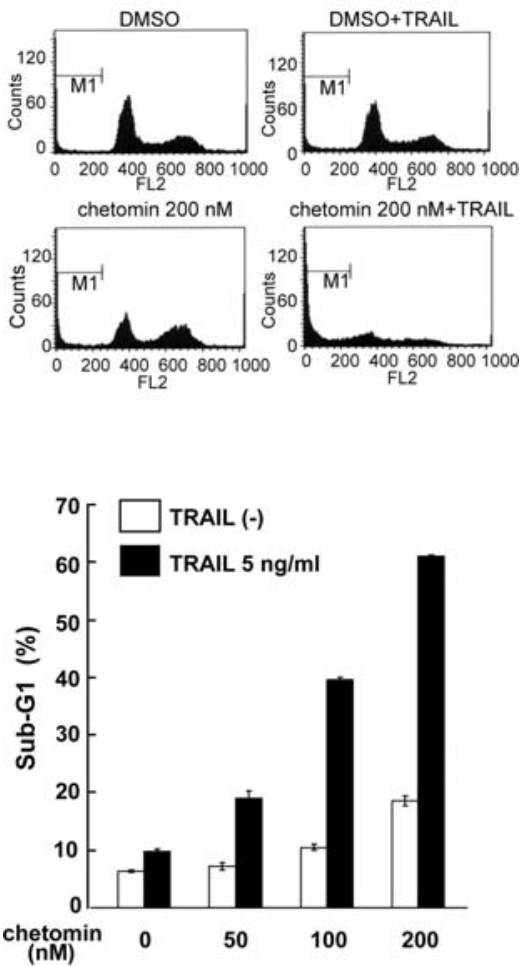

D

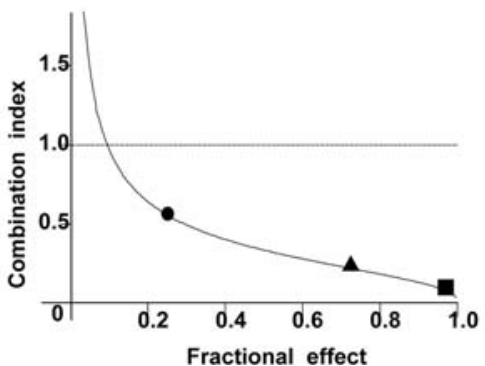

Figure 1. Chetomin sensitizes PC-3 cells to TRAIL-induced apoptosis. (A) Structural formula of chetomin. (B) Prostate cancer PC-3 cells were treated with $5 \mathrm{ng} / \mathrm{ml}$ TRAIL and/or $200 \mathrm{nM}$ chetomin and then photographed after $24 \mathrm{~h}$. (C) PC-3 cells were treated with $5 \mathrm{ng} / \mathrm{ml}$ TRAIL and the indicated concentrations of chetomin for $24 \mathrm{~h}$. The sub-G1 population (M1) was analyzed by flow cytometry. Columns, means of triplicate data; bars, SD. (D) Combination index was calculated using the CalcuSyn software. PC-3 cells were treated with TRAIL and chetomin for $24 \mathrm{~h}$ at the indicated concentrations as follows and the sub-G1 population was analyzed. $\bullet, 100 \mathrm{nM}$ chetomin and $5 \mathrm{ng} / \mathrm{ml}$ TRAIL; $\mathbf{\Delta}, 200 \mathrm{nM}$ chetomin and $10 \mathrm{ng} / \mathrm{ml} \mathrm{TRAIL} ; \mathbf{m}, 400 \mathrm{nM} \mathrm{chetomin}$ and $20 \mathrm{ng} / \mathrm{ml} \mathrm{TRAIL}$.

instructions. Total RNA $(2 \mu \mathrm{g})$ was reversely transcribed to cDNA in $20 \mu 1$ reaction volume, with MMLV-reverse transcriptase (Promega, Madison, WI), using oligo (dT) primers (TOYOBO, Osaka, Japan), according to the manufacturer's instructions. Quantitative real-time RT-PCR was carried out using an RT-PCR system GeneAmp7300 (Applied Biosystems, Foster, CA). Real-time quantitative reverse transcriptionPCR primer-probe sets for XIAP mRNA (Hs00745222_s1) and Human Euk 18S rRNA were purchased from Applied Biosystems. The expression level of XIAP mRNA was normalized against the level of $18 \mathrm{~S}$ rRNA in the same sample.

\section{Results}

Chetomin sensitizes prostate cancer PC-3 cells to TRAILinduced apoptosis. In this experiment, we used the SCADS inhibitor kit II, consisting of 96 chemical inhibitors provided by the Screening Committee of Anticancer Drugs in Japan as described in Materials and methods. For searching compounds that sensitize prostate cancer PC-3 cells to TRAIL-induced apoptosis, we treated various 96 compounds from the SCADS inhibitor kit II with $5 \mathrm{ng} / \mathrm{ml}$ TRAIL using PC-3 cells, and quantified apoptotic cells by measuring the sub-G1 population (data not shown). Consequently, we identified chetomin (Fig. 1A) as a compound which could enhance the effect of TRAIL. To confirm these data, we treated PC-3 cells with $5 \mathrm{ng} / \mathrm{ml}$ TRAIL and $200 \mathrm{nM}$ chetomin (Fig. 1B). TRAIL or chetomin treatment alone did not change the cell condition; however, combined treatment with TRAIL and chetomin killed most cells. Next, we quantified apoptotic cells by measuring the sub-G1 population. As shown in Fig. 1C, TRAIL or chetomin alone weakly induced 


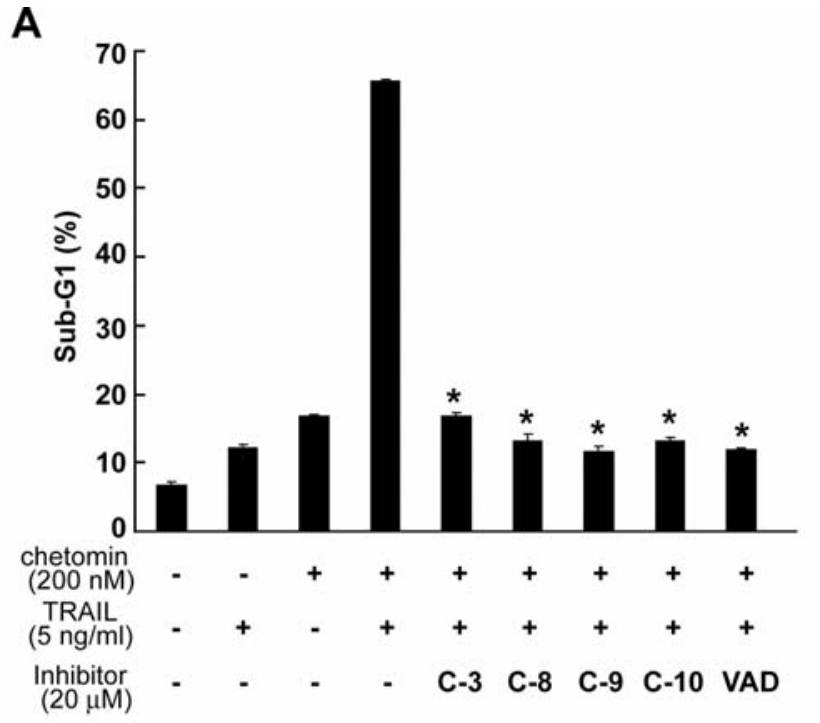

B

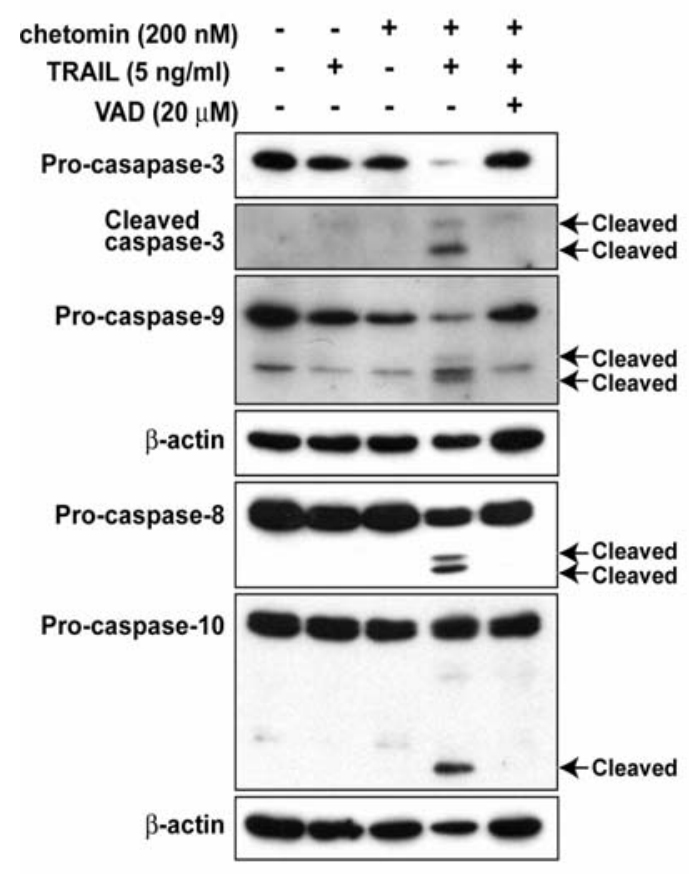

C
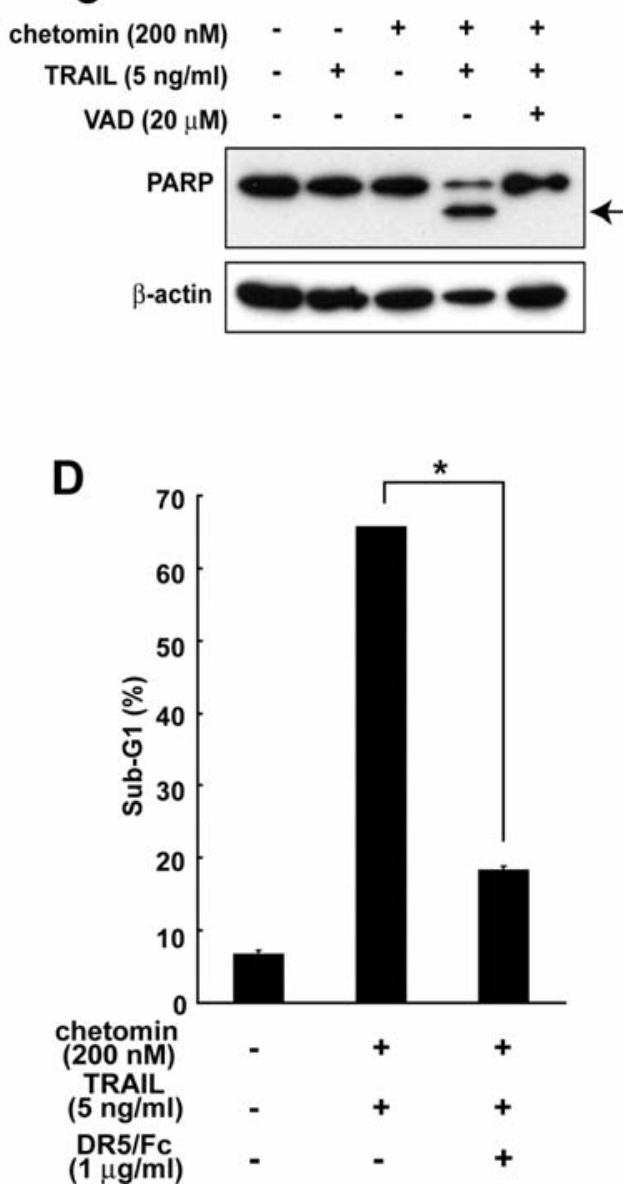

Figure 2. The combination of chetomin and TRAIL induces caspase-dependent apoptosis in PC-3 cells. (A) PC-3 cells were treated with $200 \mathrm{nM}$ chetomin, $5 \mathrm{ng} / \mathrm{ml}$ TRAIL, and/or various caspase inhibitors $(20 \mu \mathrm{M})$ for $24 \mathrm{~h}$. The sub-G1 population was analyzed by flow cytometry. Columns, means of triplicate data; bars, SD; "P<0.05. C-3, zDEVD-fmk caspase-3 inhibitor; C-8, zIETD-fmk caspase-8 inhibitor; C-9, zLEHD-fmk caspase-9 inhibitor; C-10, zAEVD-fmk caspase-10 inhibitor; VAD, zVAD-fmk pancaspase inhibitor. (B) Western blotting of caspase-3, - $8,-9,-10$. PC-3 cells were treated with $200 \mathrm{nM}$ chetomin and/or $5 \mathrm{ng} / \mathrm{ml}$ TRAIL with or without $20 \mu \mathrm{M} \mathrm{zVAD}$-fmk for $24 \mathrm{~h}$. ß-actin is a loading control. (C) Western blotting of PARP. (D) PC-3 cells were treated with $200 \mathrm{nM}$ chetomin, $5 \mathrm{ng} / \mathrm{ml}$ TRAIL, and/or $1 \mu \mathrm{g} / \mathrm{ml}$ DR5/Fc chimera protein for $24 \mathrm{~h}$. The sub-G1 population was analyzed by flow cytometry. Columns, the means of triplicate data; bars, $\mathrm{SD}$; ${ }^{*} \mathrm{P}<0.05$.

apoptosis, but the combination of chetomin and TRAIL strongly induced apoptosis. Moreover, we calculated the combination index $(\mathrm{CI})$, and found that the apoptosis induced by chetomin and TRAIL was synergistic because the CI was $<1$ (Fig. 1D). These results indicate that chetomin enhances TRAIL sensitivity in PC-3 cells. 
A
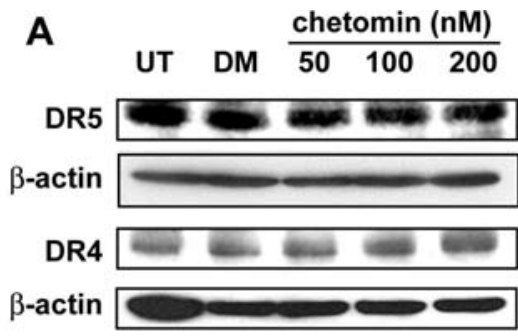

B
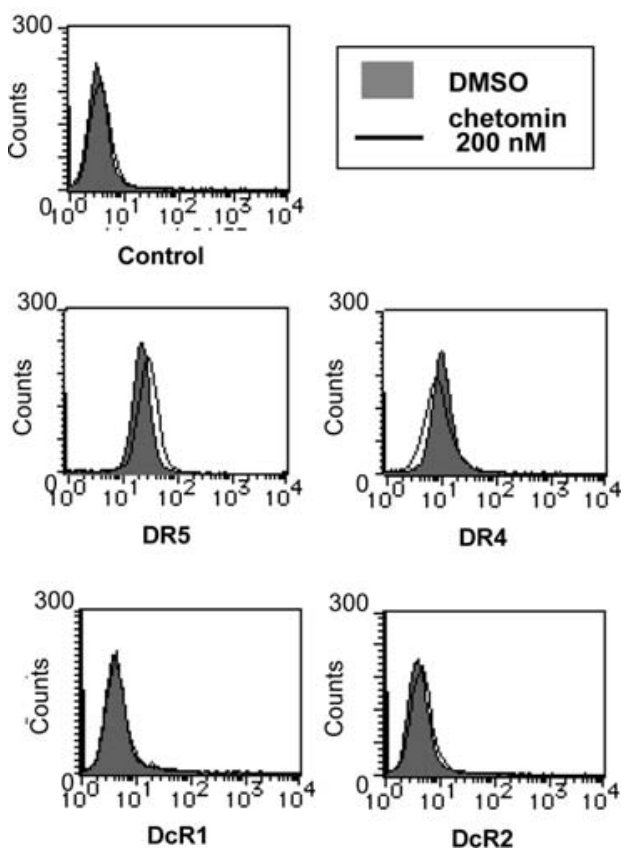

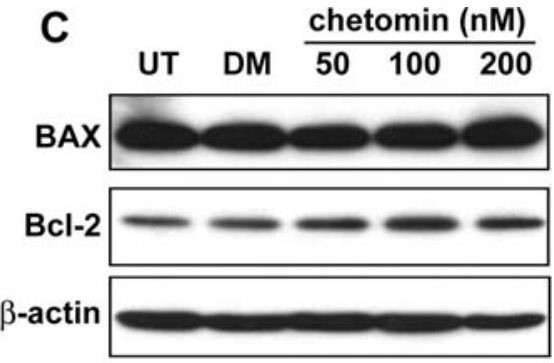

Bcl-xL $\rightleftharpoons-\infty$

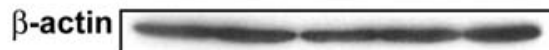

D

chetomin (nM)
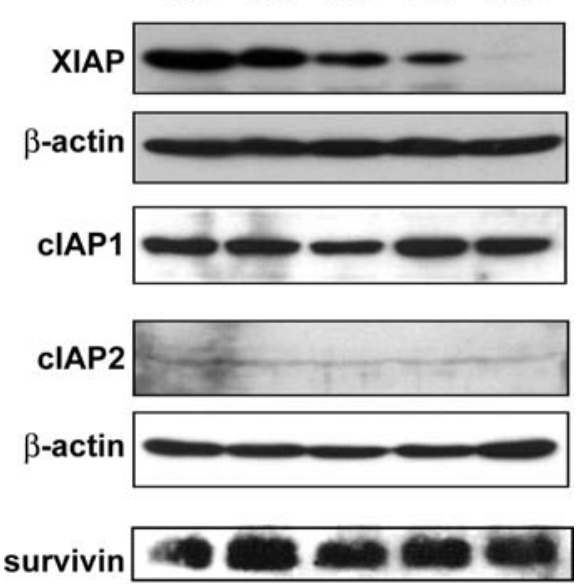

$\beta$-actin

Figure 3. Chetomin selectively down-regulates the expression of XIAP. (A) Western blotting of TRAIL receptors (DR5 and DR4). PC-3 cells were treated with the indicated concentrations of chetomin for $24 \mathrm{~h}$. B-actin is a loading control. UT, untreated; DM, treated with DMSO. (B) PC-3 cells were treated with DMSO or $200 \mathrm{nM}$ chetomin for $24 \mathrm{~h}$. Subsequently, cells were stained with isotype control IgG and monoclonal antibodies generated against the extracellular domain of TRAIL receptors DR5, DR4, DcR1 and DcR2. Data were analyzed by flow cytometry. Gray area histogram, DMSO; black line histogram, chetomin. (C) Western blotting of Bcl-2 family members (BAX, Bcl-2 and Bcl-xL). PC-3 cells were treated as shown in (A). (D) Western blotting of IAP family members (XIAP, cIAP1, cIAP2 and survivin). PC-3 cells were treated as shown in (A).

The combination of chetomin and TRAIL induces caspasedependent apoptosis in PC-3 cells. To confirm whether the apoptosis induced by chetomin and TRAIL depends on caspases, we used caspase inhibitors and analyzed sub-G1 by flow cytometry (Fig. 2A). Caspase-3, $-8,-9$ and -10 inhibitors and the pan-caspase inhibitor zVAD-fmk efficiently blocked apoptosis induced by combined treatment of chetomin and TRAIL. Additionally, we performed Western blotting of caspase-3, $-9,-8$ and -10 (Fig. 2B). Treatment with chetomin or TRAIL alone did not induce cleavage of caspases, but combined treatment with chetomin and TRAIL induced caspase cleavage. Poly (ADP-ribose) polymerase (PARP) is a substrate of caspase and is used as a marker of apoptotic cells (24). The combination of chetomin and TRAIL clearly cleaved PARP, and zVAD-fmk completely blocked cleavage, although treatment with chetomin or TRAIL alone did not cause PARP cleavage (Fig. 2C). Moreover, a dominant negative form of TRAIL receptors, DR5/Fc chimera protein, effectively blocked apoptosis induced by combined treatment with chetomin and TRAIL (Fig. 2D). These results indicate that co-treatment with chetomin and TRAIL induces caspasedependent apoptosis through the interaction between TRAIL and TRAIL receptors.

Chetomin selectively down-regulates the expression of XIAP. To elucidate the molecular mechanism of apoptosis induced by combined treatment with chetomin and TRAIL, we examined the effect of chetomin on the expression of apoptotic factors related to the TRAIL pathway. First, we examined expression levels of TRAIL receptors, DR5 and DR4 after treatment with chetomin. Chetomin did not affect DR5 and DR4 expressions (Fig. 3A). Furthermore, we examined cell- 


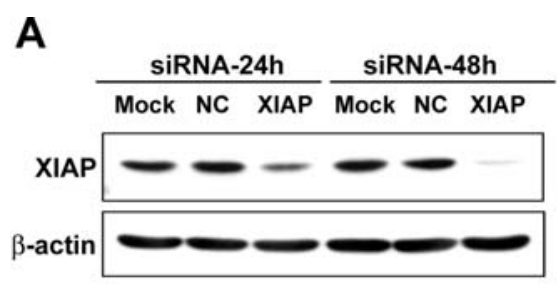

B
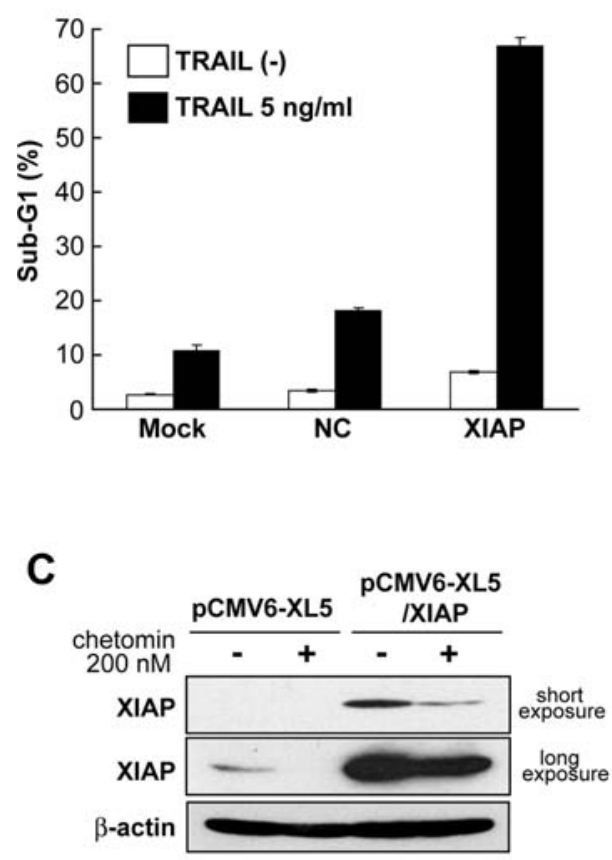

D

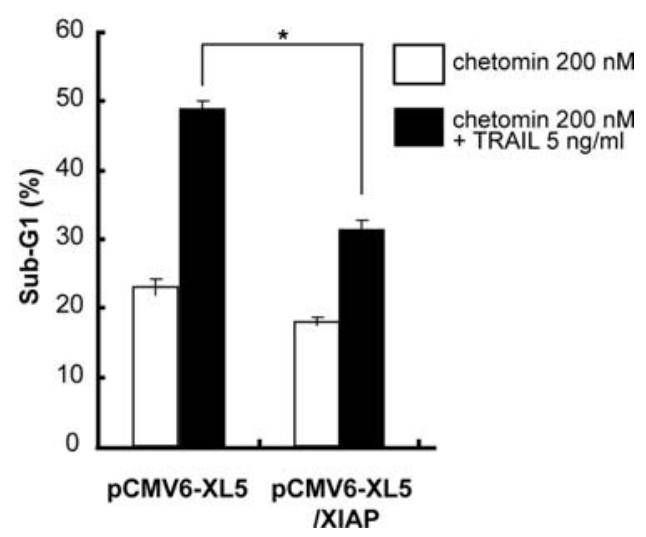

Figure 4. Down-regulation of XIAP by chetomin sensitizes PC-3 cells to TRAIL-induced apoptosis. (A) PC-3 cells were transfected with non-coding siRNA (NC), XIAP siRNA or transfection reagent alone (Mock). Twenty-four or $48 \mathrm{~h}$ after transfection, cells were harvested, and Western blotting of XIAP was performed. B-actin is a loading control. (B) PC-3 cells were transfected as shown in (A). Twenty-four hours after transfection, cells were treated with or without $5 \mathrm{ng} / \mathrm{ml}$ TRAIL. The sub-G1 population was analyzed by flow cytometry. Columns, means of triplicate data; bars, SD (C) PC-3 cells were transiently transfected with pCMV6-XL5 control or pCMV6-XL5/XIAP plasmid construct. Twenty-four hours after transfection, cells were treated with or without $200 \mathrm{nM}$ chetomin for $24 \mathrm{~h}$ and then harvested, followed by Western blotting of XIAP. (D) PC-3 cells were transfected as described in (C). Twenty-four hours after transfection, cells were treated with $200 \mathrm{nM}$ chetomin and/or $5 \mathrm{ng} / \mathrm{ml}$ TRAIL for $24 \mathrm{~h}$. The sub-G1 population was analyzed by flow cytometry. Columns, means of triplicate data; bars, $\mathrm{SD} ;{ }^{*} \mathrm{P}<0.05$. surface TRAIL receptor expression by flow cytometry. As shown in Fig. 3B, chetomin did not change cell-surface expressions of DR5, DR4, decoy receptor 1 (DcR1) and DcR2. $\mathrm{BAX}, \mathrm{Bcl}-2$ and $\mathrm{Bcl}-\mathrm{xL}$ protein levels were not affected by chetomin (Fig. 3C). As shown in Fig. 3D, treatment with chetomin markedly decreased XIAP protein levels in a dosedependent manner. However, chetomin had no effect on the expression of other IAP family members. These results suggest that down-regulation of XIAP protein by chetomin is an underlying mechanism by which chetomin sensitizes PC-3 cells to TRAIL-induced apoptosis.

Down-regulation of XIAP by chetomin sensitizes $P C-3$ cells to TRAIL-induced apoptosis. We next examined whether down-regulation of XIAP expression is involved in the sensitization of TRAIL-induced apoptosis in PC-3 cells, using XIAP siRNA instead of chetomin. The knockdown effect with XIAP siRNA was confirmed by Western blotting (Fig. 4A). Twenty-four or $48 \mathrm{~h}$ after transfection, XIAP siRNA efficiently down-regulated the expression of XIAP. As shown in Fig. 4B, we confirmed that siRNA-mediated knockdown of XIAP sensitized PC-3 cells to TRAIL-induced apoptosis. In contrast, we examined whether overexpression of XIAP contributes to the resistance to combined treatment with chetomin and TRAIL. PC-3 cells were transiently transfected with pCMV6-XL5 control or pCMV6-XL5/XIAP plasmid constructs and treated with or without $200 \mathrm{nM}$ chetomin for $24 \mathrm{~h}$. As a result, the exogenous XIAP protein as well as endogenous XIAP protein was reduced by the treatment of chetomin as shown in Fig. 4C. Moreover, the exogenous XIAP protein sufficiently but not completely suppressed the TRAIL/chetomin-induced apoptosis, suggesting that both XIAP-dependent and -independent mechanisms might exist for the activity of chetomin (Fig. 4D). These results indicate that down-regulation of XIAP by chetomin is, at least in part, a molecular mechanism of sensitization to TRAIL-induced apoptosis.

Chetomin decreases protein levels of XIAP in a proteasomedependent manner, but not $m R N A$ levels. To investigate the mechanism of XIAP down-regulation by chetomin, we performed time-course Western blotting. Treatment with chetomin induced a rapid decrease of XIAP protein levels from $2 \mathrm{~h}$ after treatment (Fig. 5A). Chetomin is known as an inhibitor of HIFs. We thus hypothesized that chetomin downregulates XIAP through HIF-dependent transcription. We examined the effect of chetomin on XIAP mRNA expression by real-time RT-PCR analysis (Fig. 5B), and found that chetomin does not decrease XIAP mRNA levels. XIAP catalyzes its own ubiquitination and is degraded by proteasomes in response to apoptotic stimuli (25), and we treated PC-3 cells with chetomin and proteasome inhibitors. As shown in Fig. 5C, chetomin-mediated XIAP down-regulation was completely prevented by MG132. Proteasome inhibitor I and proteasome inhibitor II also prevented XIAP down-regulation by chetomin. On the other hand, it has been reported that Akt can phosphorylate XIAP, and the phospho-XIAP is protected from ubiquitination and degradation (26). We then examined the effect of chetomin on the expressions of total Akt and phosphoAkt, and found that chetomin did not increase total Akt and 
A

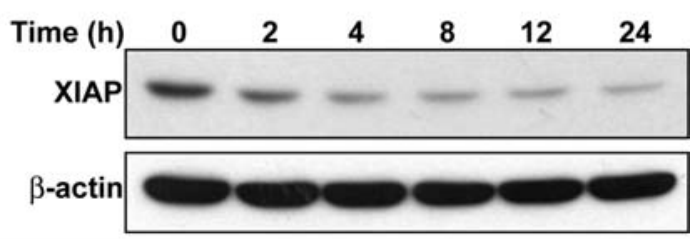

B

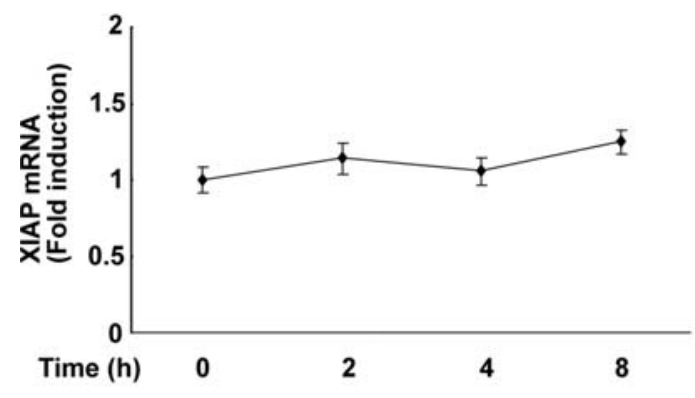

C
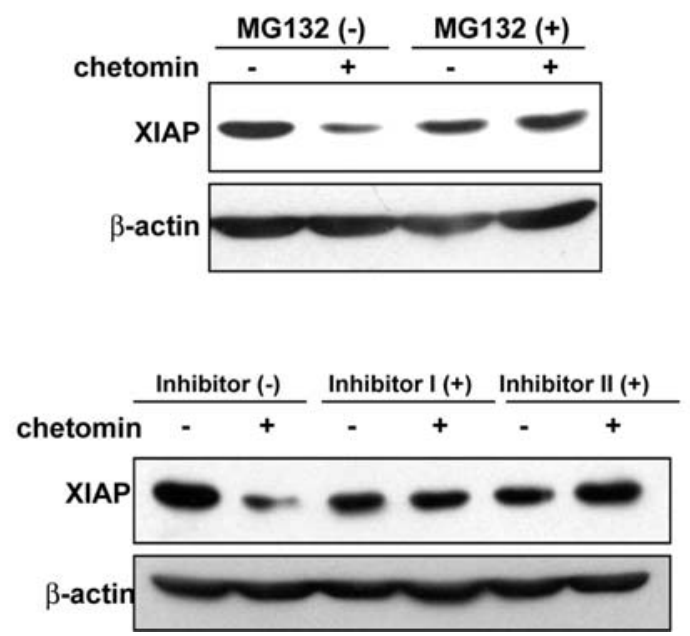

D

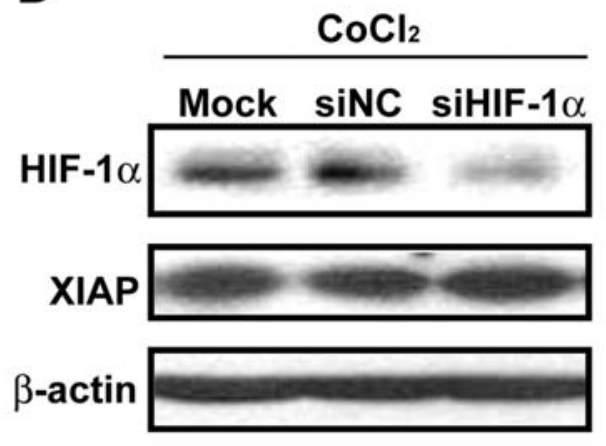

$\mathbf{E}$
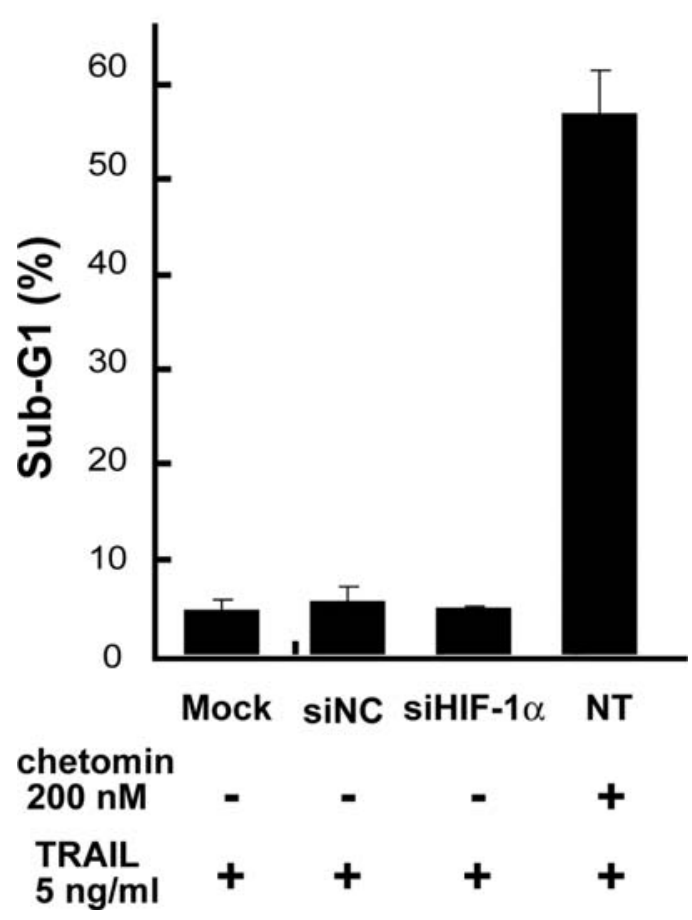

Figure 5. Chetomin does not decrease XIAP mRNA levels but protein levels in a proteasome-dependent manner. (A) PC-3 cells were treated with 200 nM chetomin for the indicated periods. Western blotting of XIAP was performed. B-actin is a loading control. (B) PC-3 cells were treated as shown in (A). XIAP mRNA levels were analyzed by real-time RT-PCR as described in Materials and methods. Points, means of triplicate data; bars, SD. (C) PC-3 cells were treated with $200 \mathrm{nM}$ chetomin and/or $5 \mu \mathrm{M}$ MG132, $10 \mu \mathrm{M}$ proteasome inhibitor I or $10 \mu \mathrm{M}$ proteasome inhibitor II for $8 \mathrm{~h}$. Western blotting of XIAP was performed. $\beta$-actin is a loading control. (D) PC-3 cells were transiently transfected with siRNA of HIF-1 $\alpha$ or a negative control for $43 \mathrm{~h}$, and the cells were treated with or without $\mathrm{CoCl}_{2}$ for $5 \mathrm{~h}$. Forty-eight hours after the transfection, cells were harvested, followed by Western blotting of HIF-1 $\alpha$ or XIAP. B-actin is a loading control. (E) PC-3 cells were transiently transfected with siRNA of HIF-1 $\alpha$ or a negative control. Twenty-four hours after the transfection, cells were treated with $5 \mathrm{ng} / \mathrm{ml}$ TRAIL with or without $200 \mathrm{nM}$ chetomin for $24 \mathrm{~h}$. The sub-G1 population was analyzed by flow cytometry. NT, non-transfected cells. Columns, the means of triplicate data; bars, SD.

very slightly decreased the phospho-Akt $8 \mathrm{~h}$ after the treatment (data not shown). In contrast, XIAP protein was drastically decreased 2-4 $\mathrm{h}$ after the treatment (Fig. 5A), strongly suggesting that decrease of XIAP by chetomin was independent of the Akt pathway. We evaluated the HIF-1 $\alpha$ dependency in the XIAP depletion and the sensitization to TRAIL-induced apoptosis by chetomin. We confirmed the effect by the down-regulation of HIF- $1 \alpha$ using HIF-1 $\alpha$ siRNA. PC-3 cells were transiently transfected with siRNA of HIF-1 $\alpha$ (Fig. 5D). The results showed that HIF-1 $\alpha$ was not involved in the sensitization to TRAIL-induced apoptosis by chetomin (Fig. 5E).
Chetomin sensitizes other urogenital cancer cells to TRAILinduced apoptosis, but not normal human PBMC. We next investigated whether chetomin also decreases XIAP protein levels in other urogenital cancer cells. As shown in Fig. 6A, XIAP protein levels were decreased by chetomin treatment in human renal cancer Caki-1 cells and human bladder cancer UM-UC-3 cells. Furthermore, we confirmed that combined treatment with chetomin and TRAIL markedly induced apoptosis in Caki-1 and UM-UC-3 cells (Fig. 6B). To examine the toxicity of the combination with chetomin and TRAIL to human normal cells, we used normal human peripheral blood mononuclear cells (PBMC) as a model. Chetomin slightly 
A

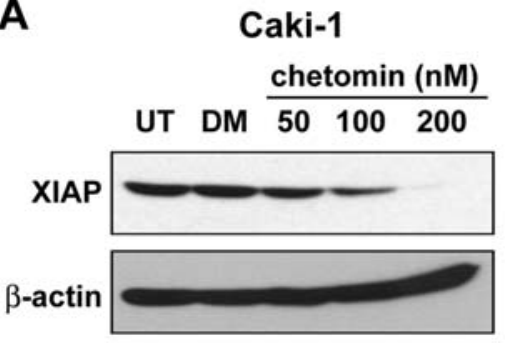

B

Caki-1

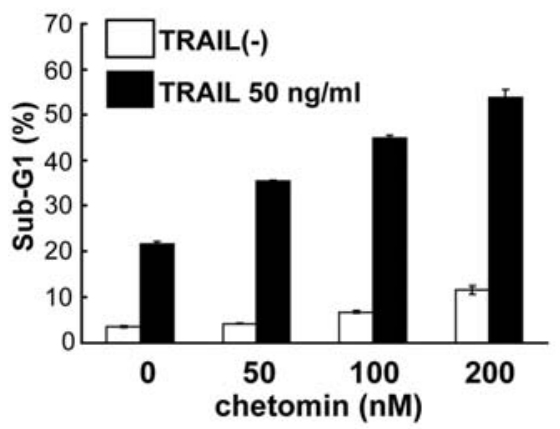

UM-UC-3

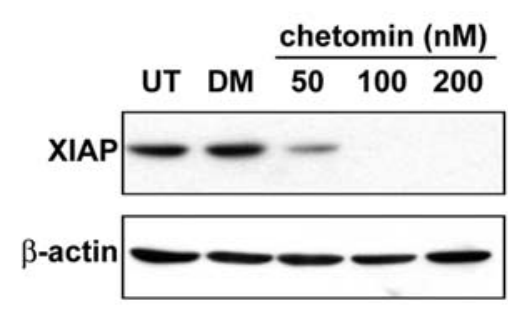

UM-UC-3

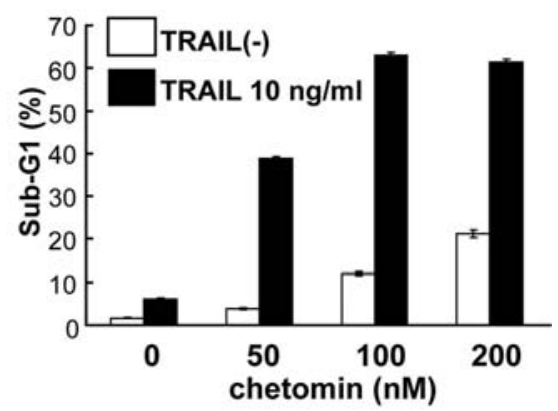

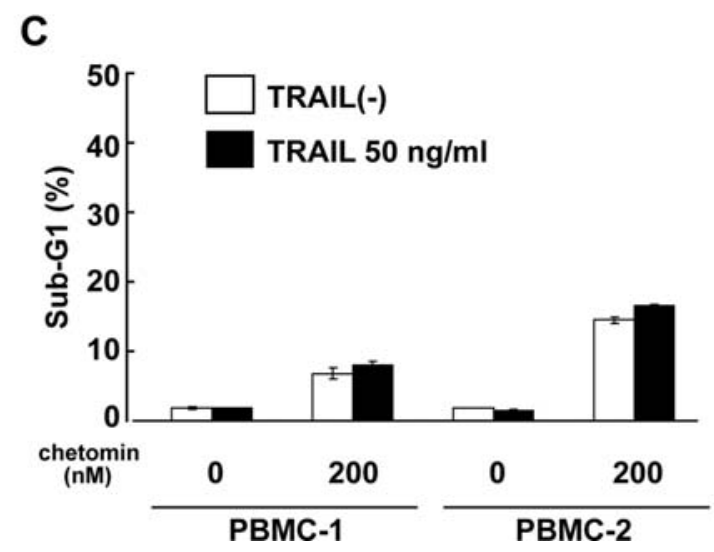

Figure 6. Chetomin does not sensitize normal human PBMC but other urogenital cancer cells to TRAIL-induced apoptosis. (A) Renal cancer Caki-1 cells and bladder cancer UM-UC-3 cells were treated with the indicated concentrations of chetomin for $24 \mathrm{~h}$. Western blotting of XIAP was performed. B-actin is a loading control. UT, untreated; DM, treated with DMSO. (B) Caki-1 cells were treated with $50 \mathrm{ng} / \mathrm{ml}$ TRAIL and the indicated concentrations of chetomin for $24 \mathrm{~h}$. UM-UC-3 cells were treated with $10 \mathrm{ng} / \mathrm{ml}$ of TRAIL and the indicated concentrations of chetomin for $24 \mathrm{~h}$. The sub-G1 population was analyzed by flow cytometry. Columns, the means of triplicate data; bars, SD. (C) Normal human PBMC derived from two different donors (PBMC-1 and -2) were treated with $50 \mathrm{ng} / \mathrm{ml}$ TRAIL and/or $200 \mathrm{nM}$ chetomin for $24 \mathrm{~h}$. The sub-G1 population was analyzed by flow cytometry. Columns, means of triplicate data; bars, SD.

induced apoptosis as a single agent in PBMC, but co-treatment of chetomin and TRAIL did not enhance apoptosis (Fig. 6C). These results suggest that the combination of chetomin and TRAIL induces apoptosis in urogenital cancer cells with little toxicity in normal cells.

\section{Discussion}

TRAIL is a promising anti-cancer agent, although some tumors remain resistant to TRAIL treatment, and a strategy to enhance TRAIL sensitivity is therefore required. In this study, we found that chetomin, known as an HIF inhibitor, enhances TRAIL sensitivity in urogenital cancer cells derived from different organs. It has been reported that deregulation of many factors related to the TRAIL pathway causes TRAIL resistance in cancer cells (11). We previously demonstrated that DR5 up-regulation is one of the strategies to enhance TRAIL sensitivity (27-29). We showed here that chetomin did not modulate TRAIL receptors (DR4, DR5, DcR1 and DcR2), Bcl-2 family proteins (Bcl-2, Bcl-xL and BAX) and 
IAP family proteins (cIAP1, cIAP2 and survivin), but chetomin specifically down-regulated XIAP proteins.

XIAP is a crucial anti-cancer target $(30,31)$, since XIAP is overexpressed in many cancer cells and is involved in a worse prognosis and early recurrence (13-15). The downregulation of XIAP by a variety of compounds has been reported (32-36). For example, flavopiridol $(32,33)$, 7-hydroxystaurosporine (32), triptolide $(34,35)$, and N,N,N',N'-tetrakis (2-pyridylmethyl) ethylenediamine (TPEN) (36) have been reported to down-regulate XIAP expression. Above all, it is noteworthy that flavopiridol (33) and triptolide (35) sensitized leukemic cells to TRAIL-induced apoptosis via the decrease of XIAP. Additionally, small molecule XIAP inhibitors (37) or UVB-induced down-regulation of XIAP (38) enhanced TRAIL-induced apoptosis. These findings built the rationale for further (pre)clinical development of XIAP inhibitors and TRAIL against cancer.

Regarding the mechanism of down-regulation of XIAP by chetomin, chetomin decreased protein levels of XIAP, but not mRNA levels (Fig. 5A and B). However, flavopiridol and triptolide reduced the expression of XIAP mRNA $(33,35)$. On the other hand, TPEN down-regulated the expression of XIAP in protein levels, but not in mRNA levels similarly to that of chetomin (36). However, the down-regulation of XIAP by TPEN could not be recovered by a proteasome inhibitor MG132, whereas chetomin-mediated down-regulation of XIAP protein was recovered by proteasome inhibitor MG132 (Fig. 5C). Therefore, the mechanism of downregulation of XIAP by chetomin is different from those of the previously reported agents.

As described above, chetomin induced the degradation of XIAP protein in a proteasome-dependent manner (Fig. 5C). The really interesting new gene (RING) domain of XIAP has E3 ubiquitin ligase activity and induces autoubiquitination and degradation of XIAP by proteasomes (25). The RING domain is also found in cIAP1 and cIAP2 but not survivin (39), but chetomin selectively down-regulates the expression of XIAP regardless of the RING domain with E3 ubiquitin ligase activity (Fig. 3D). Akt phosphorylates XIAP at residue serine-87 and phospho-XIAP by Akt is protected from ubiquitination and degradation (26). We then examined expression levels of total Akt and phospho-Akt, but chetomin had no effect on the Akt pathway (data not shown). Moreover, we considered that the function of chetomin was independent of HIF for the following reasons. i) We performed all experiments under the normoxic conditions. In normoxia, chetomin is not known to inhibit the transcription by HIF. ii) We investigated the expression of VEGF, a typical target gene of HIF, but chetomin did not alter the VEGF mRNA level (data not shown). iii) We actually evaluated the HIF-1 $\alpha$ dependency in the XIAP depletion and the sensitization to TRAILinduced apoptosis by chetomin. The results using HIF-1 $\alpha$ siRNA showed that HIF- $1 \alpha$ was not involved in the sensitization to TRAIL-induced apoptosis by chetomin (Fig. 5E).

The combination of chetomin and TRAIL induced apoptosis in urogenital cancer cells but chetomin did not enhance TRAIL-induced apoptosis in normal human PBMC (Fig. 6C). As an anti-cancer treatment, tumor-specific toxicity is required without any effects on normal cells. Thus, the combination of chetomin and TRAIL may meet this require- ment. Furthermore, down-regulation of XIAP sensitizes various cancer cell lines to anti-cancer agents in clinical applications, such as cisplatin, etoposide and docetaxel (40-42), and chetomin may also increase sensitivity to these treatment modalities as well as TRAIL.

In conclusion, we have shown a novel function of chetomin, which synergistically enhances TRAIL-induced apoptosis via down-regulation of XIAP protein expression in urogenital cancer cells.

\section{Acknowledgments}

SCADS inhibitor kit II was a kind gift from Screening Committee of Anticancer Drugs supported by Grant-in-Aid for Scientific Research on Priority Area Cancer from the Ministry of Education, Culture, Sports, Science and Technology, Japan. This work was partly supported by the Japanese Ministry of Education, Culture, Sports, Science and Technology.

\section{References}

1. Tannock IF, de Wit R, Berry WR, et al: Docetaxel plus prednisone or mitoxantrone plus prednisone for advanced prostate cancer. N Engl J Med 351: 1502-1512, 2004.

2. Motzer RJ, Hutson TE, Tomczak P, et al: Sunitinib versus interferon $\alpha$ in metastatic renal-cell carcinoma. N Engl J Med 356: 115-124, 2007.

3. Escudier B, Eisen T, Stadler WM, et al: Sorafenib in advanced clear-cell renal-cell carcinoma. N Engl J Med 356: 125-134, 2007.

4. Wiley SR, Schooley K, Smolak PJ, et al: Identification and characterization of a new member of the TNF family that induces apoptosis. Immunity 3: 673-682, 1995.

5. Ashkenazi A, Pai RC, Fong S, et al: Safety and antitumor activity of recombinant soluble Apo2 ligand. J Clin Invest 104: 155-162, 1999.

6. Keane MM, Ettenberg SA, Nau MM, Russell EK and Lipkowitz S: Chemotherapy augments TRAIL-induced apoptosis in breast cell lines. Cancer Res 59: 734-741, 1999.

7. Lawrence D, Shahrokh Z, Marsters S, et al: Differential hepatocyte toxicity of recombinant Apo2L/TRAIL versions. Nat Med 7: 383-385, 2001.

8. Pitti RM, Marsters SA, Ruppert S, Donahue CJ, Moore A and Ashkenazi A: Induction of apoptosis by Apo-2 ligand, a new member of the tumor necrosis factor cytokine family. J Biol Chem 271: 12687-12690, 1996.

9. Walczak H, Miller RE, Ariail K, et al: Tumoricidal activity of tumor necrosis factor-related apoptosis-inducing ligand in vivo. Nat Med 5: 157-163, 1999.

10. Ashkenazi A and Herbst RS: To kill a tumor cell: the potential of proapoptotic receptor agonists. J Clin Invest 118: 1979-1990, 2008.

11. Zhang L and Fang B: Mechanisms of resistance to TRAILinduced apoptosis in cancer. Cancer Gene Ther 12: 228-237, 2005.

12. Schimmer AD, Dalili S, Batey RA and Riedl SJ: Targeting XIAP for the treatment of malignancy. Cell Death Differ 13: 179-188, 2006.

13. Mizutani Y, Nakanishi H, Li YN, et al: Overexpression of XIAP expression in renal cell carcinoma predicts a worse prognosis. Int J Oncol 30: 919-925, 2007.

14. Li M, Song T, Yin ZF and Na YQ: XIAP as a prognostic marker of early recurrence of nonmuscular invasive bladder cancer. Chin Med J 120: 469-473, 2007.

15. Seligson DB, Hongo F, Huerta-Yepez S, et al: Expression of $\mathrm{X}$-linked inhibitor of apoptosis protein is a strong predictor of human prostate cancer recurrence. Clin Cancer Res 15: 6056-6063, 2007.

16. Ndozangue-Touriguine O, Sebbagh M, Merino D, Micheau O, Bertoglio J and Breard J: A mitochondrial block and expression of XIAP lead to resistance to TRAIL-induced apoptosis during progression to metastasis of a colon carcinoma. Oncogene 27: 6012-6022, 2008. 
17. Wilkinson JC, Cepero E, Boise LH and Duckett CS: Upstream regulatory role for XIAP in receptor-mediated apoptosis. Mol Cell Biol 24: 7003-7014, 2004.

18. Deveraux QL, Roy N, Stennicke HR, et al: IAPs block apoptotic events induced by caspase- 8 and cytochrome c by direct inhibition of distinct caspases. EMBO J 17: 2215-2223, 1998.

19. Chawla-Sarkar M, Bae SI, Reu FJ, Jacobs BS, Lindner DJ and Borden EC: Downregulation of Bcl-2, FLIP or IAPs (XIAP and survivin) by siRNAs sensitizes resistant melanoma cells to Apo2L/ TRAIL-induced apoptosis. Cell Death Differ 11: 915-923, 2004.

20. McManus DC, Lefebvre CA, Cherton-Horvat G, et al: Loss of XIAP protein expression by RNAi and antisense approaches sensitizes cancer cells to functionally diverse chemotherapeutics. Oncogene 23: 8105-8117, 2004.

21. Kung AL, Zabludoff SD, France DS, et al: Small molecule blockade of transcriptional coactivation of the hypoxia-inducible factor pathway. Cancer Cell 6: 33-43, 2004.

22. Nakata S, Yoshida T, Horinaka M, Shiraishi T, Wakada M and Sakai T: Histone deacetylase inhibitors upregulate death receptor 5/TRAIL-R2 and sensitize apoptosis induced by TRAIL/APO2-L in human malignant tumor cells. Oncogene 23: 6261-6271, 2004.

23. Zu K, Bihani T, Lin A, Park YM, Mori K and Ip C: Enhanced selenium effect on growth arrest by BiP/GRP78 knockdown in p53-null human prostate cancer cells. Oncogene 25: 546-554, 2006.

24. Lazebnik YA, Kaufmann SH, Desnoyers S, Poirier GG and Earnshaw WC: Cleavage of poly(ADP-ribose) polymerase by a proteinase with properties like ICE. Nature 371: 346-347, 1994.

25. Yang Y, Fang S, Jensen JP, Weissman AM and Ashwell JD Ubiquitin protein ligase activity of IAPs and their degradation in proteasomes in response to apoptotic stimuli. Science 288: 874-877, 2000.

26. Dan HC, Sun M, Kaneko S, et al: Akt phosphorylation and stabilization of X-linked inhibitor of apoptosis protein (XIAP). J Biol Chem 279: 5405-5412, 2004.

27. Goda AE, Yoshida T, Horinaka M, et al: Mechanisms of enhancement of TRAIL tumoricidal activity against human cancer cells of different origin by dipyridamole. Oncogene 29: 3435-3445, 2008.

28. Taniguchi H, Yoshida T, Horinaka M, et al: Baicalein overcomes tumor necrosis factor-related apoptosis-inducing ligand resistance via two different cell-specific pathways in cancer cells but not in normal cells. Cancer Res 68: 8918-8927, 2008

29. Yasuda T, Yoshida T, Goda AE, et al: Anti-gout agent allopurinol exerts cytotoxicity to human hormone-refractory prostate cancer cells in combination with TRAIL. Mol Cancer Res 6: 1852-1860, 2008.

30. Schimmer AD, Welsh K, Pinilla C, et al: Small-molecule antagonists of apoptosis suppressor XIAP exhibit broad antitumor activity. Cancer Cell 5: 25-35, 2004.
31. Cillessen SA, Reed JC, Welsh K, et al: Small-molecule XIAP antagonist restores caspase-9 mediated apoptosis in XIAPpositive diffuse large B-cell lymphoma cells. Blood 1: 369-375, 2008.

32. Kitada S, Zapata JM, Andreeff M and Reed JC: Protein kinase inhibitors flavopiridol and 7-hydroxy-staurosporine downregulate antiapoptosis proteins in B-cell chronic lymphocytic leukemia. Blood 96: 393-397, 2000.

33. Rosato RR, Dai Y, Almenara JA, Maggio SC and Grant S: Potent antileukemic interactions between flavopiridol and TRAIL/ Apo2L involve flavopiridol-mediated XIAP downregulation. Leukemia 18: 1780-1788, 2004.

34. Carter BZ, Mak DH, Schober WD, et al: Triptolide induces caspase-dependent cell death mediated via the mitochondrial pathway in leukemic cells. Blood 108: 630-637, 2006

35. Carter BZ, Mak DH, Schober WD, et al: Triptolide sensitizes AML cells to TRAIL-induced apoptosis via decrease of XIAP and p53-mediated increase of DR5. Blood 111: 3742-3750, 2008.

36. Makhov P, Golovine K, Uzzo RG, et al: Zinc chelation induces rapid depletion of the $\mathrm{X}$-linked inhibitor of apoptosis and sensitizes prostate cancer cells to TRAIL-mediated apoptosis. Cell Death Differ 15: 1745-1751, 2008

37. Vogler M, Walczak H, Stadel D, et al: Small molecule XIAP inhibitors enhance TRAIL-induced apoptosis and antitumor activity in preclinical models of pancreatic carcinoma. Cancer Res 69: 2425-2434, 2009.

38. Thayaparasingham B, Kunz A, Peters N and Kulms D: Sensitization of melanoma cells to TRAIL by UVB-induced and NF-KB-mediated downregulation of XIAP. Oncogene 28: 345-362, 2009.

39. Eckelman BP, Salvesen GS and Scott FL: Human inhibitor of apoptosis proteins: why XIAP is the black sheep of the family. EMBO Rep 7: 988-994, 2006

40. Sasaki H, Sheng Y, Kotsuji F and Tsang BK: Down-regulation of X-linked inhibitor of apoptosis protein induces apoptosis in chemoresistant human ovarian cancer cells. Cancer Res 60: 5659-5666, 2000.

41. Hu Y, Cherton-Horvat G, Dragowska V, et al: Antisense oligonucleotides targeting XIAP induce apoptosis and enhance chemotherapeutic activity against human lung cancer cells in vitro and in vivo. Clin Cancer Res 9: 2826-2836, 2003.

42. LaCasse EC, Cherton-Horvat GG, Hewitt KE, et al: Preclinical characterization of AEG35156/GEM 640, a second-generation antisense oligonucleotide targeting X-linked inhibitor of apoptosis. Clin Cancer Res 12: 5231-5241, 2006. 